\title{
Hereditary Deficiency of Lactate Dehydrogenase H-Subunit
}

\author{
Hajime WaKabayashi, Masao Tsuchiya, Ken Yoshino, Kohei KaKu* and Hiroshi Shigei
}

We report herein the fifth family of hereditary deficiency of lactate dehydrogenase (LDH) Hsubunit with an autosomal recessive inheritance including two cases of complete deficiency. Their LDH activities were low both in the serum and in the red blood cells (RBC). Electrophoretic analysis revealed that the patients with the complete deficiency had only the $\mathrm{LDH}_{5}$ isozyme. The complete deficiency was associated with marked elevation of fructose-1,6-diphosphate (FDP) and dihydroxyacetonephosphate (DHAP) and a less marked rise in glyceraldehyde-3-phosphate (GA3P) among glycolytic intermediates in the RBC. Furthermore, hemolysis was observed in the present cases, but this finding was not included in the other reports.

(Internal Medicine 35: 550-554, 1996)

Key words: autosomal recessive inheritance, complete deficiency, fructose-1,6-diphosphate, dihydroxyacetonephosphate, glyceraldehyde-3-phosphate, hemolysis

\section{Introduction}

Lactate dehydrogenase (LDH) exists in a tetrameric form composed of $\mathrm{H}$-subunits and $\mathrm{M}$-subunits in varying proportions in different tissues. Thus, there are five LDH isozymes, all of which have different affinity to pyruvate (1).

LDH deficiency is classified into H-subunit deficiency and M-subunit deficiency (2). To date, four cases of complete deficiency of LDH H-subunit have been detected (2) and two of these cases have already been reported $(3,4)$. Maekawa et al (5) estimated the gene frequency of the complete deficiency of $\mathrm{H}$ subunit (squared number of gene frequency) to be $6.31 \times 10^{-7}$, about one person per one million. The complete deficiency of M-subunit (6) is characterized by myoglobinuria after severe exercise. An ischemic work causes a marked increase in the blood levels of pyruvate and in rhabdomyolysis. In addition, some patients develop erythematosquamous skin lesions which become worse in the summer (7). No laboratory values are abnormal including serum LDH activity when the patients are in the resting state. Only after severe exercise, M-subunit deficiency manifests marked elevation of FDP, DHAP and GA3P in the muscle cells. Conversely, $\mathrm{H}$-subunit deficiency shows no clinical symptoms even though the serum LDH activity is low.

In the present paper, we report two cases of the complete deficiency of $\mathrm{H}$-subunit and their family and compare these to the two cases of complete deficiencies already reported.

\section{Materials and Methods}

\section{Case reports}

Case 1

A 49-year-old male visited our hospital presenting pain in his right foot. He had had urine sugar and proteinuria for the previous 10 years. A seventy five gram glucose tolerance test (75-g GTT) revealed that he had diabetes mellitus. To control his diabetes, he was admitted to our hospital. The laboratory data was notable for the extremely low level of LDH activity (Table 1). Furthermore, indirect hyperbilirubinemia and reticulocytosis were observed (Table 1), which were indicative of hemolysis. Twenty-four-hour creatinine clearance $(54.4 \mathrm{ml} /$ $\min$ ) and hyperuricemia (Table 1) revealed diabetic nephropathy. Other examinations including a load electrocardiogram were within normal limits. The right foot pain was due to achillobursitis.

\section{Case 2}

This patient is the 46-year-old, younger brother of case 1 (Fig. 1). His serum LDH activity was also very low (Table 1). Various tests including a 75-g GTT were almost within normal limits except for the indirect hyperbilirubinemia and reticulocytosis (Table 1). The family history disclosed the consanguineous marriage of their parents (Fig. 1). Their grandmothers are in sisterhood.

From the Department of Internal Medicine, Shigei Hospital, Kurashiki and *the Third Department of Internal Medicine, Yamaguchi University Medical School, Ube

Received for publication July 5, 1995; Accepted for publication April 4, 1996

Reprint requests should be addressed to Dr. Hajime Wakabayashi, the First Department of Internal Medicine, Okayama University Medical School, 2-5-1 Shikatacho, Okayama 700 
Table 1. Laboratory Data of Two Patients with Complete Deficiency of LDH H-Subunit

\begin{tabular}{lrrrc}
\hline & Normal range & Case 1 & Case 2 \\
\hline Complete blood count & & & & \\
\hline White blood cell count $(\times 100 / \mu 1)$ & $50-80$ & 64 & 44 \\
Red blood cell count $(\times 10,000 / \mu \mathrm{l})$ & $380-530$ & 490 & 485 \\
Hemoglobin $(\mathrm{g} / \mathrm{dl})$ & $12-18$ & 14.8 & 14.9 \\
Hematocrit $(\%)$ & $35-48$ & 41.8 & 44.0 \\
Platelet count $(\times 10,000 / \mu \mathrm{l})$ & $12-28$ & 22.5 & 20.0 \\
Reticulocyte percentage $(\%)$ & $0.3-1.1$ & $\underline{1.7}$ & $\underline{1.4}$ \\
Haptoglobin $(\mathrm{mg} / \mathrm{dl})$ & $41-273$ & 105 & not tested \\
\hline
\end{tabular}

Blood chemistry

\section{Zinc sulfate turbidity test}

$$
\text { (Kunkel U) }
$$

Aspartate aminotransferase $(\mathrm{U} / l)$

Alanine aminotransferase $(\mathrm{U} / l)$

Lactate dehydrogenase $(\mathrm{U} / l)$

Alkaline phosphatase $(\mathrm{U} / l)$

Leucine aminopeptidase $(\mathrm{U} / l)$

$\gamma$-Glutamyl transpeptidase (U/l)

Total bilirubin (mg/dl)

Direct bilirubin $(\mathrm{mg} / \mathrm{dl})$

Uric acid (mg/dl)

Blood urea nitrogen $(\mathrm{mg} / \mathrm{dl})$

Creatinine (mg/dl)

Triglyceride (mg/dl)

Total cholesterol (mg/dl)

Fast blood sugar (mg/dl)

$\begin{array}{rlll}4 & -12 & 6.5 & 11.1 \\ 10 & -27 & 19 & 21 \\ 5 & -33 & 23 & 28 \\ 180-460 & \underline{36} & \underline{42} \\ 6-50 & 22.9 & 20.7 \\ 30-70 & 49 & 75 \\ 4-63 & 20 & 12 \\ 0.2-0.8 & \underline{1.35} & \underline{1.61} \\ 0.1-0.3 & 0.28 & 0.44 \\ 2.9-6.5 & \underline{8.6} & 3.6 \\ 6.0-17 & 18 & 14 \\ 0.7-1.7 & 1.0 & 0.8 \\ 50-105 & 160 & 43 \\ 12-220 & 226 & 186 \\ 70-110 & \underline{185} & 102\end{array}$

\section{Procedure}

Sera and red blood cells (RBC) were collected from case 1, case 2 , their family, two healthy volunteers as normal controls and two patients with diabetes mellitus. One of the two diabetic patients (DM patient 1) is a 57-year-old female with mild diabetes mellitus without complication. The other one (DM patient 2) is a 55-year-old male, who has severe diabetes mellitus, controlled with subcutaneous injection of intermediate type insulin, and diabetic nephropathy.

\section{Assay of serum $\mathrm{LDH}$ activities}

The serum LDH activity was assayed according to the method of Wróblewski and LaDue (8) using a 7150 type autoanalyzer (Hitachi, Tokyo, Japan). It was then converted into the international units. The final concentration of the reagents (HA testwako; Wako Pure Chemical Ind., Osaka, Japan) for the assay were as follows: pyruvate $4.0 \mathrm{mM}$, nicotinamide adenine dinucleotide (NADH) $0.27 \mathrm{mM}$, and Tris-buffer $10 \mathrm{mM}$ ( $\mathrm{pH} \mathrm{9.0).} \mathrm{The} \mathrm{serum} \mathrm{LDH} \mathrm{activity} \mathrm{was}$ expressed in terms of $\mathrm{U} / l$ at $37^{\circ} \mathrm{C}$.

\section{Detection of LDH isozymes}

$\mathrm{LDH}$ isozymes in the serum and in the RBC were detected

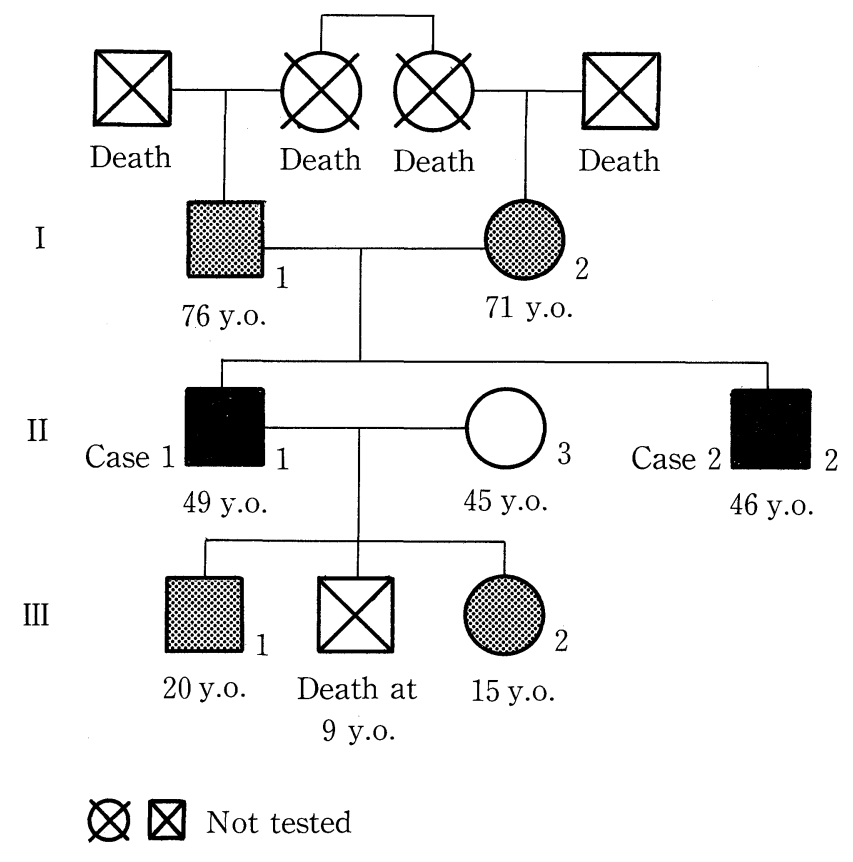

Figure 1. Pedigree of the fifth family of deficiency of $\mathrm{LDH} \mathrm{H}$ subunit. These cases seem to be inherited in the form of autosomal recessive as in the previous two cases. Case 1 and Case 2 are considered to be homozygous, while their parents and children are considered to be heterozygous. Black squares indicate homozygotes, and shaded squares and circles indicate heterozygotes. Abbreviations are as follows: I-1: father, I-2: mother, II-1: case 1 (arrow), II-2: case 2, II-3: wife of case 1, III-1: son of case 1, III-2: daughter of case 1 .

by cellulose acetate electrophoresis according to the method of Shioya et al (9). The final concentration of reagents (Taitan; Helena Laboratories, Saitama, Japan) for the detection were: D,L-Lactate, $120 \mathrm{mM}$; NAD ${ }^{+}, 5 \mathrm{mM}$; 3-(4,5-dimetyl-2triazolyl)-2,5-diphenyl-2 $\mathrm{H}$-tetrazolium bromide, $2 \mathrm{mM}$; nitrotetrazolium blue, $0.6 \mathrm{mM}$; and Tris- $\mathrm{HCl}, 50 \mathrm{mM}$ (pH 7.98.1).

Assay of the activities of glycolytic enzymes and glycolytic intermediates in the $R B C$

The activities of glycolytic enzymes in the RBC including $\mathrm{LDH}$ were assayed according to the method of Beutler (10) using the photodensitometer U-3200 (Hitachi). Glycolytic intermediates and adenine nucleotides were measured according to the method described by Minakami et al (11), using the photodensitometer U-3200. The LDH activity in the RBC was expressed in terms of $\mathrm{U} / \mathrm{g}$ hemoglobin $(\mathrm{Hb})$ at $37^{\circ} \mathrm{C}$.

\section{Results}

Both case 1 and case 2 had extremely low LDH activities in the serum and in the RBC (Fig. 2). Furthermore, they had only one $\mathrm{LDH}$ isozyme, $\mathrm{LDH}_{5}$, both in the serum and in the $\mathrm{RBC}$ 


\section{WAKABAYASHI et al}
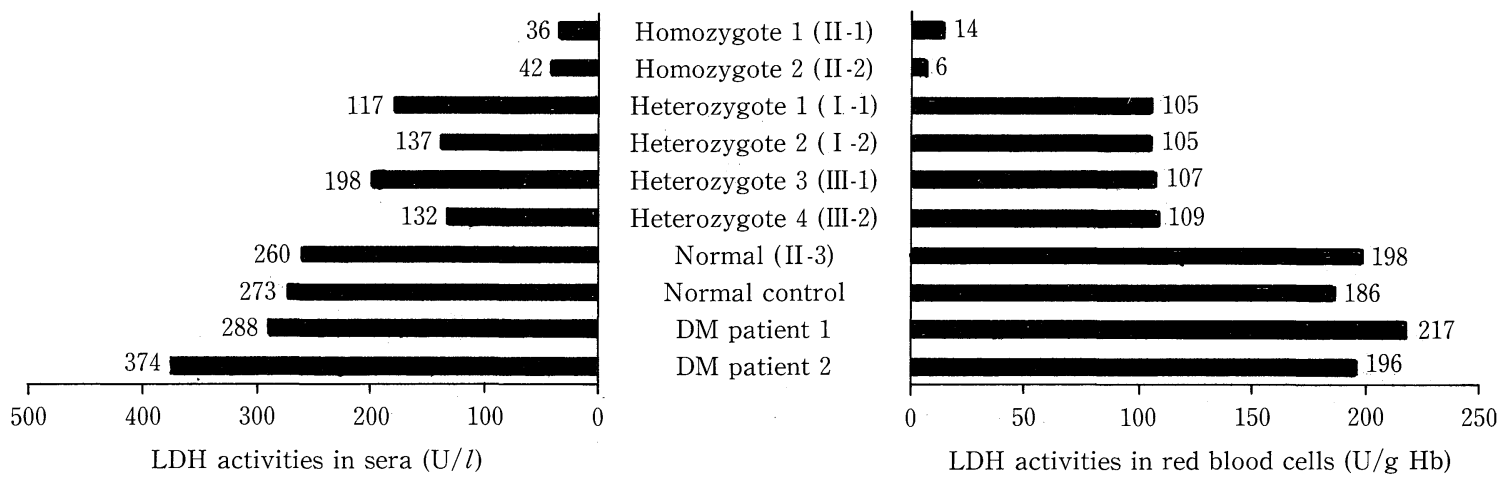

Figure 2. LDH activities in sera and red blood cells. The normal ranges of the LDH activity in the serum and in the $\mathrm{RBC}$ are from 180 to $480 \mathrm{U} / l$ and from 184 to $240 \mathrm{U} / \mathrm{g} \mathrm{Hb}$, respectively. Numbers for the corresponding columns show the levels of $\mathrm{LDH}$ activity.

(Fig. 3). Their parents and the daughter of case 1 had slightly low serum LDH activities, between $70 \%$ of the normal lower limit and the normal lower limit (Fig. 2). Defective isozyme was not observed in the patterns of LDH isozymes of their parents. In $\mathrm{RBC}$, the $\mathrm{LDH}$ activities of the family of case 1 were approximately half of the normal control. Regarding the glycolytic intermediates of the RBC, the fructose-1, 6-diphosphate (FDP) and dihydroxyacetonephosphate (DHAP) levels were very high in case 1 and case 2 (Table 2). However, the glyceraldehyde-3-phosphate (GA3P) levels were not as high as in case 1 and case 2 (Table 2). None of their family members showed elevated levels of these intermediates. All other glycolytic enzyme activities or glycolytic intermediates in RBC were within the normal limits. The mixed test ruled out the existence of inhibitors against the expression of LDH activity in the serum (12). Regarding glucose metabolism, there were no notable differences between the two patients with diabetes mellitus and the normal control.

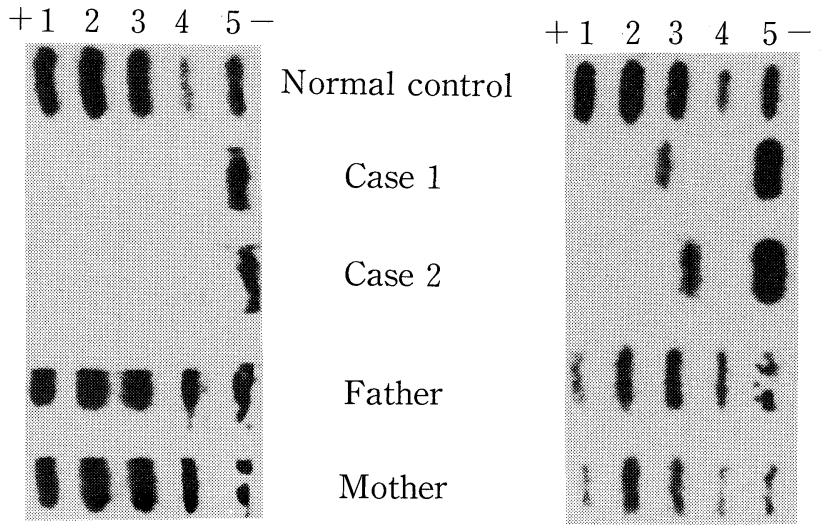

Figure 3. LDH isozyme patterns obtained from electrophoresis of sera and of the hemolysate. The left image depicts the former which were electrophoresed within 24 hours after sera were collected and the right depicts the latter. In the right image, the bands which correspond to LDH3 isozyme in case 1 or in case 2 , are hemoglobin.

Table 2. Comparison of FDP, DHAP and ATP Levels among H-Subunit Deficiencies, Normal Control and Patients with Diabetes Mellitus

\begin{tabular}{lcccc}
\hline & $\begin{array}{c}\text { FDP } \\
(\mathrm{nmol} / \mathrm{ml} \text { RBC})\end{array}$ & $\begin{array}{c}\text { DHAP } \\
(\mathrm{nmol} / \mathrm{ml} \text { RBC})\end{array}$ & $\begin{array}{c}\text { GA3P } \\
(\mathrm{nmol} / \mathrm{ml} \mathrm{RBC})\end{array}$ & $\begin{array}{c}\text { ATP } \\
(\mathrm{nmol} / \mathrm{ml} \mathrm{RBC})\end{array}$ \\
\hline Normal range & $6.0-18.3$ & $11.2-21.6$ & $2.6-9.4$ & $1,010-1,550$ \\
Homozygote 1 (II-1) & $\underline{57.8}$ & $\underline{179.4}$ & 9.7 & 1,177 \\
Homozygote 2 (II-2) & $\underline{138.3}$ & $\underline{199.3}$ & 11.8 & 930 \\
Heterozygote 1 (I-1) & 3.2 & 23.7 & 5.0 & 1,220 \\
Heterozygote 2 (I-2) & 18.8 & 4.8 & 5.8 & 1,212 \\
Heterozygote 3 (III-1) & 10.8 & 1.8 & 9.2 & 1,168 \\
Heterozygote 4 (III-2) & 1.8 & 14.6 & 2.0 & 1,197 \\
Normal (II-3) & 2.8 & 22.7 & 4.4 & 1,036 \\
Normal control & 7.1 & 18.1 & 8.5 & 1,116 \\
DM patient 1 & 8.5 & 18.3 & 4.8 & 1,239 \\
DM patient 2 & 6.8 & 20.6 & 8.5 & 1,336 \\
\hline
\end{tabular}




\section{Complete Deficiency of LDH H-Subunit}

\section{Discussion}

All of the cases of LDH H-subunit deficiency reported to date have been in Japan. It is possible, therefore, that the Hsubunit deficiency is characteristic of the Japanese race. The first and second cases of complete deficiency of LDH H-subunit were reported by Kitamura et al (3) in 1971, and by Joukyuu et al (4) in 1989, respectively. These two cases were both from nonconsanguineous marriage. The present family includes two complete deficiencies. This implies that the consanguineous marriage brings about this disease in a high probability. The first case was reported to have diabetes mellitus. However, none of the members of the family of either the first or the second case had any previous disease. Similarly, the present cases and their family had no previous disease except that case 1 had diabetes mellitus. There is nothing known connecting diabetes mellitus to $\mathrm{H}$-subunit deficiency. Although $\mathrm{H}$-subunit deficiency shows no clinical symptoms, myoglobinuria occurs after severe exercise in cases of M-subunit deficiency (5). The difference of the clinical feature between $\mathrm{H}$-subunit and M-subunit deficiency appears to depend on the affinity to pyruvate of the remaining LDH isozyme. M-subunit has a much higher affinity to pyruvate than does the H-subunit (1). M-subunit rich isozymes such as $\mathrm{LDH}_{5}$, mainly exist in the liver or the muscle which performs vigorous glycolysis. The defect of the high affinity isozyme in these tissues can lead to various clinical symptoms.

The following findings related to glucose metabolism of the $\mathrm{RBC}$ in patients with $\mathrm{H}$-subunit deficiency are unique: 1) Marked accumulation of pyruvate is a predicted consequence of low LDH activity; however, this accumulation is within the normal limits. 2) The levels of FDP or DHAP are considerably higher than the level of GA3P. 3) At the oxidation of GA3P, glycolysis is obviously obstructed and the production of ATP must be slowed down as a consequence. However in the present cases, the ATP values were not decreased so much (Table 2). 4) With regard to the heterozygote, the levels of FDP and DHAP did not show a rising tendency. These findings can be explained as follows: Glycolysis in erythrocytes always terminates in lactate, because mitochondriae are absent. Hence, if NADH is reoxidized in reducing methemoglobin to hemoglobin, the endproduct of glucose metabolism is pyruvate. If NADH is not reoxidized, pyruvate is reduced in the LDH step, forming lactate as the final end-product (13). The lactate or pyruvate formed diffuses out of the RBC (14). Thus the accumulation of pyruvate or lactate dose not occur.

In glycolysis, three irreversible reactions exist and the reaction with the greatest effect in this disease is: 1) Fructose-6phosphate $\rightarrow$ FDP. Under physiological condtions, LDH catalyzes the following reaction: pyruvate $+\mathrm{NADH}+\mathrm{H}^{+} \rightarrow$ lactate $+\mathrm{NAD}^{+}$. NAD produced in this reaction is used to oxidize GA3P to 1,3-diphosphoglycerate (1,3-DPG). If LDH activity is very low, the reoxidation of NADH is delayed. The deficiency of NAD causes the delay of oxidation from GA3P to $1,3-\mathrm{DPG}$, which is coupled with this reaction. In addition, at equilibrium, $96 \%$ of the triose phosphate is DHAP (1). As a result, the heavy accumulation of DHAP and FDP is inevitably provoked and reaction 1) expedites it further. That is the reason why in patients homozygous for the deficiency of the LDH Hsubunit, the levels of FDP and DHAP preferentially elevate to the level of GA3P in RBC.

In glucose metabolism of erythrocytes, about 5 to 10 percent of the glucose utilized, passes pentose phosphate shunt before reentering the main stream of glycolysis (15). In this shunt, ATP is not consumed. Probably this shunt is used more in the RBC in cases of deficiency of LDH H-subunit.

In heterozygous patients, the activities of $\mathrm{LDH}_{1}$ and $\mathrm{LDH}_{2}$ in RBC were about half of the normal controls, and the activities of $\mathrm{LDH}_{3}, \mathrm{LDH}_{4}$ and $\mathrm{LDH}_{5}$ were almost equal to those of the normal controls (Table 3). The major difference between the homozygous and the heterozygous was whether $\mathrm{LDH}_{3}$ and $\mathrm{LDH}_{4}$ remain or not, which possess high affinity to pyruvate. If these isozymes are not related to the maintenance of normal reoxidation of NADH to NAD, the levels of FDP or DHAP must increase in the heterozygous, because the activity of $\mathrm{LDH}_{1}$ and $\mathrm{LDH}_{2}$ is only half of the normal activity. Probably, in heterozygotes, the remaining $\mathrm{LDH}_{3}$ and $\mathrm{LDH}_{4}$ isozymes manage to aide the reoxidation of NADH to NAD. To elucidate this point will help clarify the role of $\mathrm{H}$-subunit in glucose metabolism in RBC.

The present two cases showed indirect hyperbilirubinemia and reticulocytosis that suggest hemolysis. Whereas hemolysis is found in almost all of the hereditary enzymatic deficiencies of erythrocytes (15), in deficiencies of LDH H-subunit, such a finding has not been reported yet. The hemolysis appears to be mild and not to reduce the serum haptoglobin level (Table 1). Light microscopic examination of the smear preparation of peripheral blood did not demonstrate an abnormality of the RBC. The Coombs' test ruled out the involvement of an

Table 3. Comparison of the LDH Activities in the RBC among Normal Control, Heterozygotes and Homozygotes

\begin{tabular}{lccccc}
\hline & $\begin{array}{c}\mathrm{LDH}_{1} \\
(\mathrm{U} / \mathrm{g} \mathrm{Hb})\end{array}$ & $\begin{array}{c}\mathrm{LDH}_{2} \\
(\mathrm{U} / \mathrm{g} \mathrm{Hb})\end{array}$ & $\begin{array}{c}\mathrm{LDH}_{3} \\
(\mathrm{U} / \mathrm{g} \mathrm{Hb})\end{array}$ & $\begin{array}{c}\mathrm{LDH}_{4} \\
(\mathrm{U} / \mathrm{g} \mathrm{Hb})\end{array}$ & $\begin{array}{c}\mathrm{LDH}_{5} \\
(\mathrm{U} / \mathrm{g} \mathrm{Hb})\end{array}$ \\
\hline Normal control & 65.1 & 66.9 & 40.9 & 7.4 & 5.6 \\
Heterozygote 1 (I-1) & 26.3 & 36.8 & 31.5 & 7.4 & 3.1 \\
Heterozygote 2 (I-2) & 21.0 & 35.7 & 33.6 & 10.5 & 4.2 \\
Homozygote 1 (II-1) & 0 & 0 & 0 & 0 & 14.0 \\
Homozygote 2 (II-2) & 0 & 0 & 0 & 0 & 6.0 \\
\hline
\end{tabular}




\section{WAKABAYASHI et al}

antibody related to hemolysis. However, it is possible that the chronic hyper-FDP or DHAP condition affects the RBC to cause hemolysis. We are now investigating this symptom in terms of the red cell life span.

Acknowledgements: We thank Prof. K. Taketa (Department of Public Health, Okayama University Medical School) and Dr. Ukita (First Department of Internal Medicine, Okayama University Medical School) for many stimulating discussions and thoughtful critiques of the manuscript, and we are grateful to Ms. Y. Kora (Technician of Third Department of Internal Medicine, Yamaguchi University Medical School), Mr. T. Kaneda and Ms. M. Tsunetoh (Director and Staff of Laboratory, Shigei Hospital).

\section{References}

1) Stryer L. Biochemistry. San Francisco: W H Freeman, 1981, ed 2, 225.

2) Kanno T. Lactate dehydrogenase subunits deficiencies. Nippon Rinshou 51 Suppl: 1060, 1993.

3) Kitamura M, Iijima N, Hashimoto F, Hiratsuka A. Hereditary deficiency of subunit $\mathrm{H}$ of lactate dehydrogenase. Clin Chim Acta 34: 419, 1971.

4) Joukyuu R, Mizuno S, Amakawa T, Tsukada T, Nishina T, Kitamura M. Hereditary complete deficiency of lactate dehydrogenase $\mathrm{H}$-subunit. Clin Chem 35: 687, 1989.

5) Maekawa M, Kanda S, Sudo K, Kanno T. Estimation of the gene frequency of lactate dehydrogenase subunit deficiencies. Am J Hum
Genet 36: 1204, 1984.

6) Kanno T, Sudo K, Takeuchi I, et al. Hereditary deficiency of lactate dehydrogenase M-subunit. Clin Chim Acta 108: 267, 1980.

7) Yoshikuni K, Tagami H, Yamada M, Sudo K, Kanno $T$. Erythematosquamous skin lesion in hereditary lactate dehydrogenase $\mathrm{M}$ subunit deficiency. Arch Dermatol 122: 1420, 1986.

8) Wróblewski F, LaDue JS. Lactic dehydrogenase activity in blood. Proc Soc Exp Bio Med 90: 210, 1955.

9) Shioya M, Yanagisawa M, Kawamura T, Kanno T. Separation of lactate dehydrogenase isoenzymes by cellulose acetate (Cellogel). Rinsho Byori (Jpn J Clin Pathol) 19: 469, 1971.

10) Beutler E. A Manual of Biochemical Methods, Grune \& Stratton, New York, 1975 ed 2

11) Minakami K, Suzuki C, Saito T, Yoshikawa H. Studies on erythrocyte glycolysis. I. Determination of the glycolytic intermediates in human erythrocytes. J Biochem (Tokyo) 58: 543, 1965.

12) Maekawa M, Sudo K, Iwahara K, Kanno T. Lactate dehydrogenase inhibition by immunoglobulin $\mathrm{G}$ in human serum. Clin Chem 32: 1347, 1986.

13) Williams WJ, Beutler E, Erslev AJ, Rundles RW. Hematology. McGrawHill, New York, 1977 ed 2.

14) Miwa S, Nishina T, Kakehashi Y, Kitamura M, Hiratsuka A, Shizume K. Studies on erythrocyte metabolism in a case with hereditary deficiency of H-subunit of lactate dehydrogenase. Acta Haematol Jpn 34: 228, 1971.

15) Jaffe ER. Hereditary hemolytic disorders and enzymatic deficiencies of human erythrocytes. Blood 35: 116, 1970. 